\title{
Molecular targeted and immune checkpoint therapy for advanced hepatocellular carcinoma
}

\author{
Ziyu Liu' ${ }^{1 \dagger}$, Yan Lin ${ }^{2 \dagger}$, Jinyan Zhang ${ }^{2}$, Yumei Zhang ${ }^{2}$, Yongqiang Li ${ }^{2}$, Zhihui Liu ${ }^{2}$, Qian Li ${ }^{2}$, Ming Luo ${ }^{2}$, \\ Rong Liang ${ }^{2^{*}}$ (D) and Jiazhou $\mathrm{Ye}^{3^{*}}$
}

\begin{abstract}
Molecular targeted therapy for advanced hepatocellular carcinoma (HCC) has changed markedly. Although sorafenib was used in clinical practice as the first molecular targeted agent in 2007, the SHARPE and Asian-Pacific trials demonstrated that sorafenib only improved overall survival (OS) by approximately 3 months in patients with advanced HCC compared with placebo. Molecular targeted agents were developed during the 10-year period from 2007 to 2016, but every test of these agents from phase II or phase III clinical trial failed due to a low response rate and high toxicity. In the 2 years after, 2017 through 2018, four successful novel drugs emerged from clinical trials for clinical use. As recommended by updated Barcelona Clinical Liver cancer (BCLC) treatment algorithms, lenvatinib is now feasible as an alternative to sorafenib as a first-line treatment for advanced HCC. Regorafenib, cabozantinib, and ramucirumab are appropriate supplements for sorafenib as second-line treatment for patients with advanced HCC who are resistant, show progression or do not tolerate sorafenib. In addition, with promising outcomes in phase II trials, immune PD-1/PD-L1 checkpoint inhibitors nivolumab and pembrolizumab have been applied for HCC treatment. Despite phase III trials for nivolumab and pembrolizumab, the primary endpoints of improved OS were not statistically significant, immune PD-1/PD-L1 checkpoint therapy remains to be further investigated. This review summarizes the development and progression of molecular targeted and immune-based checkpoint therapies in HCC.
\end{abstract}

Keywords: Hepatocellular carcinoma, Molecular targeted therapy, Immunotherapy

\section{Introduction}

Hepatocellular carcinoma (HCC) is the sixth most common malignancy and the fourth leading cause of cancer-related death worldwide [1]. Because the symptoms of early HCC are often inconspicuous, most patients are diagnosed at an advanced stage, eliminating the possibility of local treatment, such as curative hepatic resection, tumor ablation or transtarterial therapy. Therefore, the systematic treatment of advanced HCC is of great concern. Since sorafenib was approved as the first small oral molecular targeted medicine for patients with advanced-

\footnotetext{
*Correspondence: ronglianggx@foxmail.com; yejiazhou2019@163.com 'Ziyu Liu and Yan Lin contributed equally to this work.

${ }^{2}$ Department of Medical Oncology, Guangxi Medical University Cancer Hospital, Nanning, Guangxi 530021, People's Republic of China

${ }^{3}$ Department of Hepatobiliary Surgery, Guangxi Medical University Cancer

Hospital, Nanning, Guangxi 530021, People's Republic of China

Full list of author information is available at the end of the article
}

stage HCC in 2007, molecular targeted therapy for advanced HCC has changed markedly. However, although the SHARPE trial (in Europe and USA) [2] and the AsianPacific study (in Asia-Pacific regions) [3] demonstrated that sorafenib significantly improved the survival benefit for patients with advanced HCC, anticancer efficacy remains unsatisfactory because sorafenib only prolongs the overall survival (OS) period by approximately 3 months compared with placebo. From 2007 to 2016, various molecular targeted drugs for advanced HCC were developed (Fig. 1, Table 1). However, most of the phase II or III clinical trials for these medicines failed, as the results did not show that these drugs achieved a better survival benefit for advanced HCC patients compared with sorafenib or they were not well tolerated with severe adverse events. Fortunately, there has been substantial progress in testing new and efficacious systemic therapies 


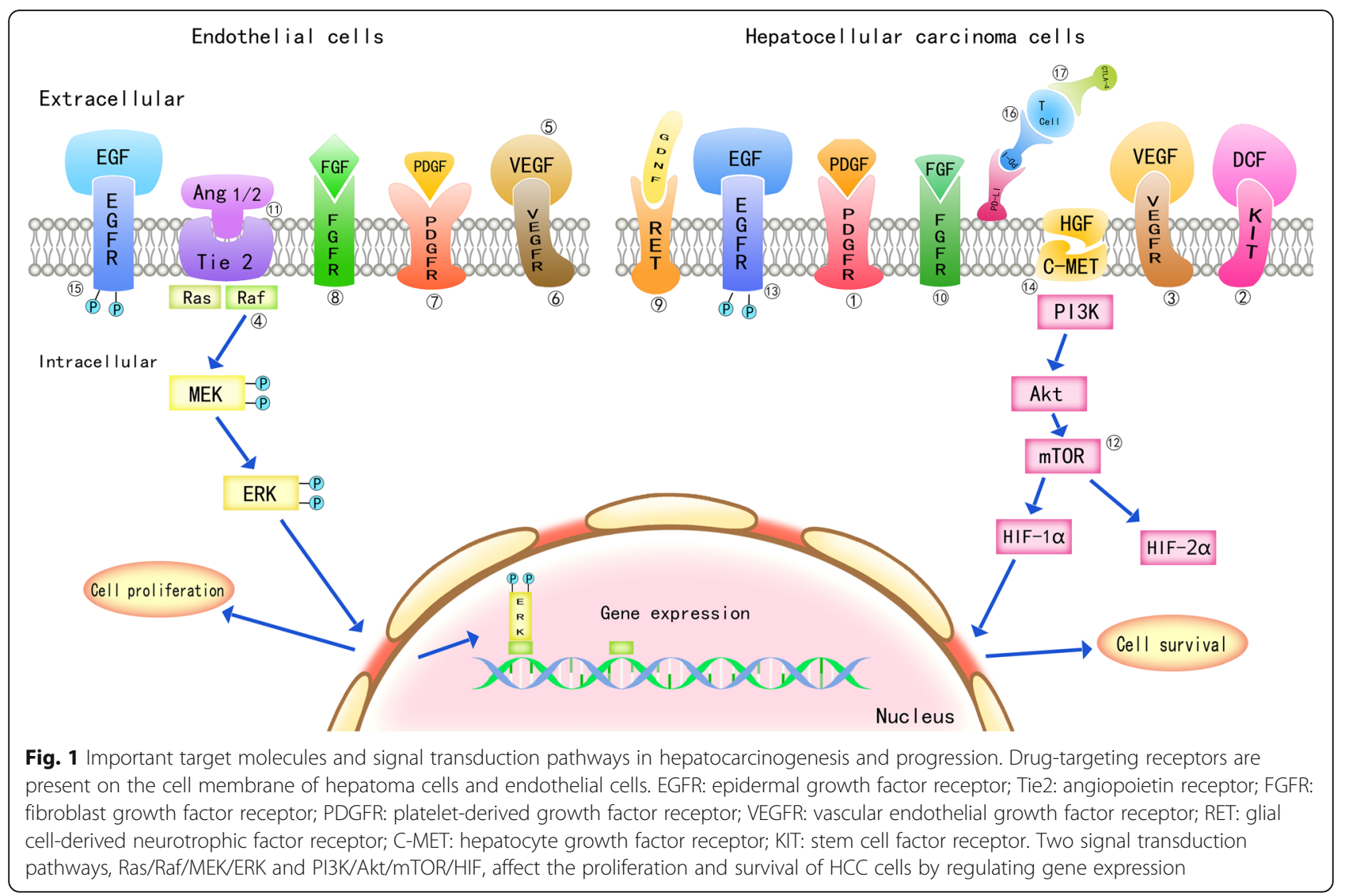

for patients with an advanced-stage HCC, with six new agents exhibiting clinical efficacy in phase 3 trials in the past 2 years. Lenvatinib has successfully become the firstline treatment in clinical practice, and regorafenib, cabozantinib, and ramucirumab have been recommended as second-line treatment options. In addition, the clinical benefits of immune-based therapies for HCC have been emerging. In a single-group phase $1 / 2$ trial [4], the novel programmed cell death 1 (PD-1) checkpoint inhibitor nivolumab resulted in promising survival in patients who had disease progression or unacceptable adverse effects with sorafenib, which prompted FDA approval under the accelerated program. In contrast, another PD-1 inhibitor, pembrolizumab, for second-line treatment did not confer longer OS or progression-free survival (PFS) compared to placebo in a recently reported phase III trial [5]. This review summarizes the development and progression of molecular targeted and immune-based checkpoint therapies in HCC.

\section{First-line systemic therapy Sorafenib}

Sorafenib is an oral small molecule multikinase inhibitor that exerts an anticancer effect by simultaneously suppressing angiogenesis via inhibition of vascular endothelial growth factor receptor (VEGFR-1,2,3) and platelet-derived growth factor receptor (PDGFR) and the growth of tumor cells directly through downregulation of the Ras/Raf/Mek/Erk signaling pathway [6, 7].

In 2007, two phase III randomized, multicenter, double-blind, placebo-controlled trials, the SHARP trial (in Europe and the USA) [2] and ORIENTAL trial (in Asia-Pacific regions) [3], reported promising results that sorafenib significantly increased survival for advanced HCC patients with different territories when compared with placebo. The SHARP trial enrolled 602 advanced HCC patients in northern America and western Europe, and the results demonstrated that the survival benefits from sorafenib were superior to placebo. The median OS was 10.7 months in the sorafenib group (a dose of $400 \mathrm{mg}$ twice daily) and 7.9 months in the placebo group. The ORIENTAL trial enrolled advanced HCC 271 patients from the Asia-Pacific region and reported a magnitude of survival benefit similar to that of the SHARP trial. The median OS was 6.5 months in patients treated with sorafenib (a dose of $400 \mathrm{mg}$ twice daily) compared with 4.2 months in those who received placebo. Based on the results from the SHARP and ORIENTAL trials, sorafenib was approved by the US FDA and EMEA for advanced HCC systematic treatment. Furthermore, in 2010, sorafenib was recommended by Barcelona Clinical Liver Cancer (BCLC) 


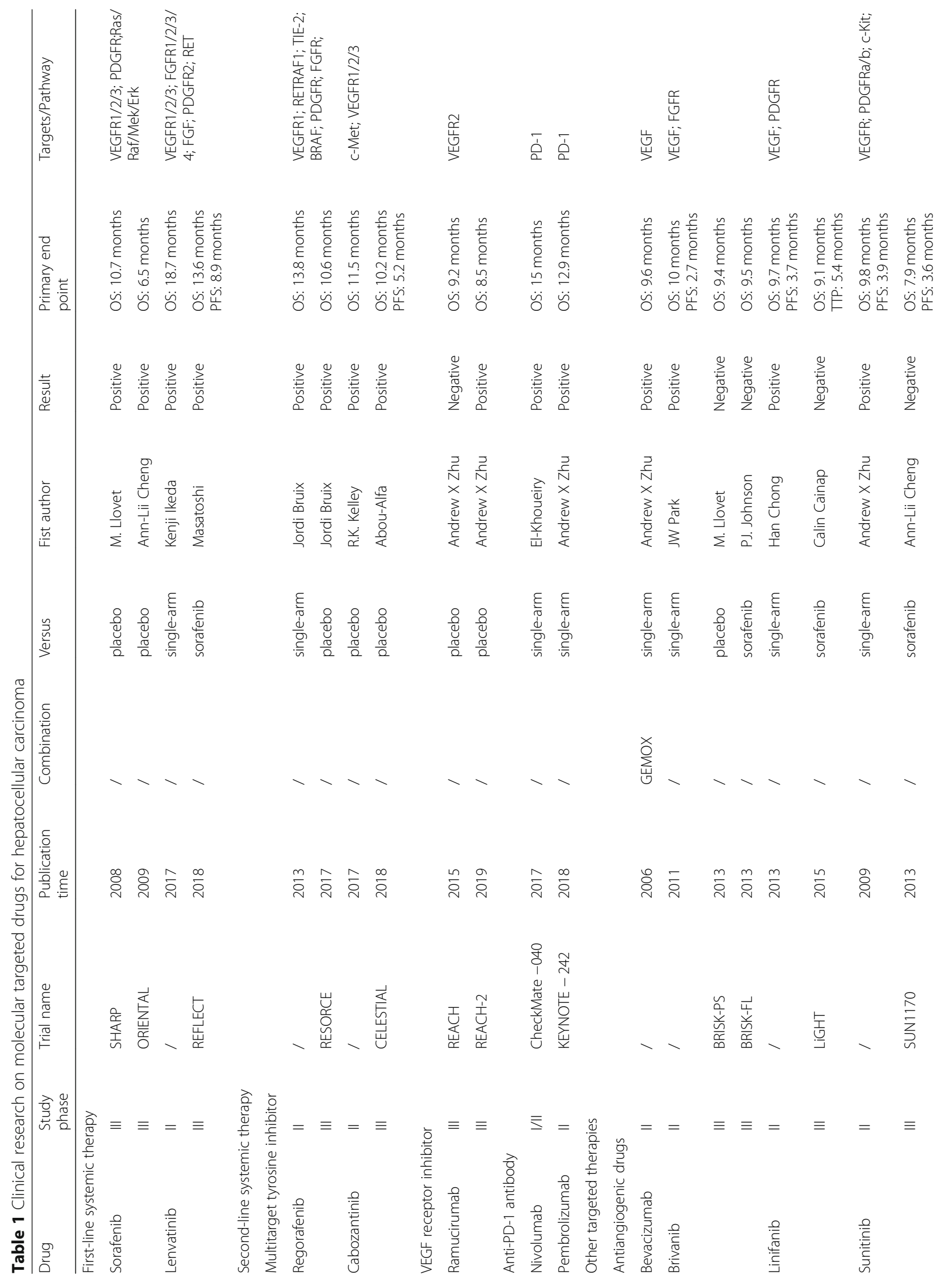




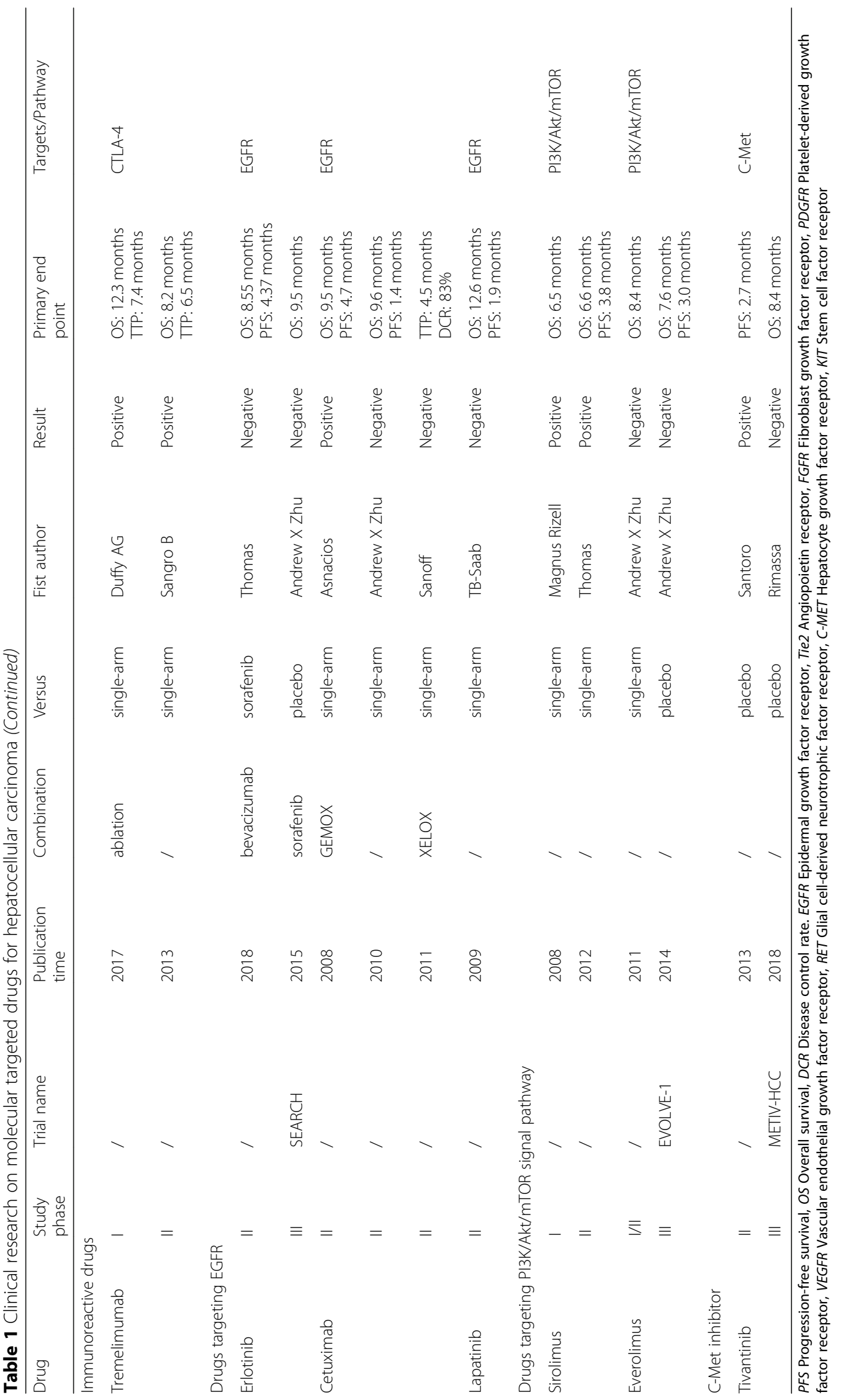


treatment algorithms [8] and version 1.2008 NCCN guidelines [9] as a first-line targeted molecular therapy for advanced HCC globally. Nonetheless, the SHARP and ORIENTAL trials reported outcomes that sorafenib only prolongs the OS period by approximately 3 months in patients with advanced HCC. Systemic therapy for advanced HCC has developed markedly since sorafenib was applied to the treatment for advanced HCC in 2007. Although many agents were developed between 2007 and 2016, most of them failed in clinical trials, and rare molecular drugs have become the 1st line and 2nd line systemic treatments for advanced HCC in clinical practice.

\section{Lenvatinib}

Lenvatinib is another oral small molecule multikinase inhibitor that selectively inhibits tyrosine kinases (e.g., VEGFR1, VEGFR2, VEGFR3), fibroblast growth factor receptor (FGFR1, FGFR2, FGFR3, FGFR4), PDGFR2, FGF and RET to suppress tumor angiogenesis and growth [10]. Lenvatinib has been certified to invoke strong antiangiogenic and anticancer effects and has been approved for the treatment of differentiated thyroid carcinoma [11]. The phase II trial [12] of lenvatinib for the treatment of patients with advanced HCC demonstrated that 12-mg QD of the agent had significant survival benefits, with a disease control rate (DCR) of $78 \%$ and a median OS of 18.7 months, as well as acceptable toxicity profiles without severe adverse events. A phase III randomized, multicenter, openlabel, non-inferiority trial, the REFLECT trial [13] enrolled 954 patients and compared the efficacy of lenvatinib versus sorafenib for first-line treatment of patients with unresectable HCC. The results presented a positive outcome, whereby lenvatinib achieved a better OS benefit than did sorafenib. The median OS duration was 13.6 months for 478 patients in the lenvatinib group ( $12 \mathrm{mg} /$ day for bodyweight $\geq 60 \mathrm{~kg}$ or $8 \mathrm{mg} /$ day for bodyweight $<60 \mathrm{~kg}$ ) compared with 12.3 months for 476 patients in the sorafenib group (400 $\mathrm{mg}$ twice-daily). In OS subanalysis, patients were stratified by race (Asian or non-Asian), vascular invasion and/or EHS (yes or no), Eastern Cooperative Oncology Group performance status (PS) (0 or 1), and body weight $(<60 \mathrm{~kg}$ or $\geq 60 \mathrm{~kg})$, and lenvatinib also resulted in longer OS than sorafenib in almost all subgroups. In particular, patients in the serum AFP level $>200 \mathrm{ng} / \mathrm{mL}$ group treated with lenvatinib had significantly longer OS than did those treated with sorafenib (10.4 months vs 8.2 months). In addition, the REFLECT trial demonstrated a statistically significant improvement compared with sorafenib with respect to all secondary efficacy endpoints, such as PFS, TTP, and ORR. The median PFS for lenvatinib was longer than that for sorafenib, and the median time to progression was 8.9 months (95\% CI 7.4-9.2) for patients in the lenvatinib group compared to 3.7 months (3.65.4) for patients in the sorafenib group. On August 16, 2018, the US FDA officially approved lenvatinib for advanced HCC systematic treatment. Moreover, lenvatinib is recommended by the version 2.2019 NCCN guidelines [14] as the second first-line targeted molecular treatment for advanced HCC.

\section{Second-line systemic therapy Multitarget tyrosine inhibitors Regorafenib}

Regorafenib is a small molecule multitarget inhibitor of VEGFR1, TIE-2, RETRAF-1, BRAF, PDGFR, FGFR, and CSF1R. In 2013, a multicenter, open-label and phase II clinical trial [15] revealed that the use of regorafenib as a second-line therapy during the progression of intermediate and advanced HCC after sorafenib treatment failure achieved promising therapeutic outcomes, with a DCR of $72 \%$ and an OS of 13.8 months, providing evidence of antitumor activity in patients with intermediate or advanced HCC that progressed following first-line sorafenib treatment. The RESORCE study [16] led by Bruix J, a randomized, double-blind, placebo-controlled, phase 3 trial, enrolled 573 patients with HCC who tolerated and progressed on sorafenib ( $\geq 400 \mathrm{mg} /$ day for $\geq 20$ of last 28 days of treatment) from 152 medical institutions in 21 countries. The participants were randomly assigned by $2: 1$ to a regorafenib (oral dose $160 \mathrm{mg}$ daily during weeks $1-3$ of each 4-week cycle) or placebo-control (once daily during weeks 1-3 of each 4-week cycle) group. The results showed that the median OS of the regorafenib group was 10.6 months, which was significantly superior to the 7.8 months observed in the placebo-control group. Considering the promising evidence from the RESORCE study, on December 22, 2017, US FDA officially approved regorafenib for use in patients with HCC. Furthermore, the version 1.2017 NCCN guidelines [17] recommend regorafenib as a second-line agent for $\mathrm{HCC}$ who progress on sorafenib treatment.

\section{Cabozantinib}

Cabozantinib is a tyrosine kinase inhibitor of c-Met, AXL, VEGFR1, - 2, and-3. A phase II trial [18] placebo-controlled randomized discontinuation study of cabozantinib in HCC patients led by R. K. Kelley randomized 12 patients to placebo and 10 to cabozantinib. The results revealed a median week 12 PFS of 5.2 months and a median week 12 OS of 11.5 months for cabozantinib, indicating good antitumor activity in HCC. Abou-Alfa et al. then carried out a randomized, double-blind, phase III clinical study [19] to evaluate 
cabozantinib as a second-line therapy for advanced HCC and the development of resistance to sorafenib, in which 707 patients were randomly assigned in a 2:1 ratio to receive cabozantinib (60 $\mathrm{mg}$ once daily) or matching placebo. The results showed significantly longer OS and PFS with cabozantinib than with placebo. The median OS and PFS were 10.2 months and 5.2 months in the cabozantinib group compared with 8.0 months and 1.9 months in the placebo group. Cabozantinib has been approved by the US FDA for the treatment of HCC. Furthermore, the NCCN guidelines [14] (version 2.2019) recommend cabozantinib as a second-line agent for HCC patients who progress on sorafenib, offering an alternative option for second-line treatment of HCC.

\section{VEGF receptor inhibitors Ramucirumab}

Ramucirumab is a recombinant IgG1 monoclonal antibody and VEGF receptor- 2 antagonist that has been approved by the US FDA for the treatment of gastric cancer [20] (on April 21, 2014), non-small cell lung cancer (on December 12, 2014) and colorectal cancer [21] (on April 29, 2015). The REACH trial was a randomized, double-blind, multicenter (154 centers in 27 countries), phase 3 trial [22] led by Andrew X. Zhu in 2010 to investigate ramucirumab versus placebo as a second-line treatment in 565 patients with advanced progressing HCC following first-line therapy with sorafenib. The results showed an OS of 9.2 months in the ramucirumab group ( $8 \mathrm{mg} / \mathrm{kg}$ every 2 weeks) compared with 7.6 months in the placebo group. Although the OS periods between the two groups were not statistically significant, in subgroup analysis, patients with elevated baseline serum AFP concentrations of $400 \mathrm{ng} / \mathrm{mL}$ or greater achieved a better OS benefit from ramucirumab compared with placebo. The median OS in the ramucirumab group was 7.8 months, which was significantly greater than the 4.2 months in the placebocontrol group. Based on this finding, the REACH-2 study [23], a randomized, double-blind, placebo-controlled, phase 3 trial also led by Andrew X. Zhu, was consequently conducted in 2015 with 292 patients with advanced $\mathrm{HCC}$ and $\alpha$-fetoprotein concentrations of $400 \mathrm{ng} / \mathrm{mL}$ or higher from 92 hospitals, clinics, and medical centers in 20 countries. Among the patients, 197 were randomly assigned to the ramucirumab group and 95 to the placebo group. According to the results, the OS period was 8.5 months in the ramucirumab group ( $8 \mathrm{mg} / \mathrm{kg}$ every 2 weeks) compared with 7.3 months in the placebo group, and the median PFS was significantly increased in the ramucirumab group ( 2.8 months) compared with the placebo group (1.6 months), though the proportion of patients' ORR was not significantly different between the groups. In addition, ramucirumab was well tolerated with a low incidence of adverse events and a manageable safety profile. Considering that the REACH-2 study confirmed the result of subgroup analysis in the REACH trial, which is the first positive phase III trial performed in a biomarker-selected patient population with advanced HCC, on May 10, 2019, the FDA approved ramucirumab as a single agent for $\mathrm{HCC}$ in patients who have an AFP $\geq 400 \mathrm{ng} / \mathrm{mL}$ and have been previously treated with sorafenib. Ramucirumab is also recommended by the NCCN guideline [14] (version 2.2019) as a potential well-tolerated second-line treatment for patients with advanced HCC and elevated AFP levels.

\section{Anti-PD-1 antibody \\ Nivolumab}

Nivolumab, a PD-1 inhibitor, was approved by the FDA in 2017 as a second-line treatment for advanced $\mathrm{HCC}$ in the presence of sorafenib resistance. A phase $1 / 2$, open-label, non-comparative, dose escalation and expansion trial [4] (CheckMate 040) led by ElKhoueiry $\mathrm{AB}$ was conducted to assess the safety and efficacy of nivolumab as a first-line therapy in patients with advanced HCC. The results revealed that in the dose-escalation phase, the overall objective response rate was $15 \%$, with a DCR of $58 \%$ and an OS of 15 months. In the dose-expansion phase, more than 200 patients who were treated with nivolumab had a sixmonth survival rate of $83 \%$ and a nine-month survival rate of $74 \%$. The study also shows that nivolumb has a manageable safety profile. On June 24, 2019, the Bristol-Myers Squibb Company published a phase 3 randomized, multicenter study evaluating opdivo (nivolumab) versus sorafenib as a first-line treatment in patients with unresectable HCC. However, per the pre-specified analysis, statistical significance for its primary endpoint of OS was not achieved, and the specific data have not been published. Regardless, the trial CheckMate-459 revealed a clear trend of improvement in OS for patients treated with opdivo compared to sorafenib, and exploration of opdivo in HCC will continue.

\section{Pembrolizumab}

Pembrolizumab is a recombinant monoclonal human immunoglobulin IgG4 antibody specific for the human PD-1 checkpoint. The FDA approved pembrolizumab for the treatment of patients with unresectable or metastatic melanoma in 2019. A non-randomized, multicenter, open-label phase II study [24] (KEYNOTE-224) led by Andrew X Zhu was performed to assess the efficacy and safety of pembrolizumab in patients with advanced HCC. In this study, participants received $200 \mathrm{mg}$ pembrolizumab intravenously every 3 weeks 
for approximately 2 years or until disease progression or unacceptable toxicity. The results showed a complete response rate of $1 \%$, partial response rate of $16 \%$, and stable rate of $44 \%$. Tumor remission rates of $17 \%$ and DCRs of more than $60 \%$ were achieved with pembrolizumab in patients with advanced HCC and were maintained for a long period of time, with a median OS of 12.9 months. In addition, pembrolizumab was well tolerated with few adverse reactions. Considering that pembrolizumab is effective and tolerable in patients with advanced HCC who had previously been treated with sorafenib, the US FDA has approved the priority review application for pembrolizumab for the indication of a second-line treatment for HCC in 2019 and progressed the KEYNOTE-240 trial [5] (a randomized, placebo-controlled phase III study of pembrolizumab vs best support care in patients with previously treated advanced HCC). Unfortunately, failure was declared for the KEYNOTE-240 trial 3 months later. Although the significance of the trial did not reach the prespecified statistical criteria, pembrolizumab reduced the risk of death by $22 \%$ and improved PFS compared with placebo. Additionally, the ORR in the pembrolizumab arm was consistent with that of KEYNOTE224 , and the safety profile was comparable to that established for pembrolizumab monotherapy. These results are consistent with KEYNOTE-224, further supporting pembrolizumab as second-line treatment for HCC patients.

\section{Other targeted therapies \\ Antiangiogenic drugs}

Because angiogenesis has been demonstrated to be a major mechanism contributing to malignant tumor growth and metastasis, antiangiogenic drugs have become an important strategy for the systematic treatment of cancers, particularly for HCC, which is a typical blood-rich tumor overexpressing various angiogenic factors.

\section{Bevacizumab}

Bevacizumab is a monoclonal antibody against VEGF. When specifically binding to VEGF, it prevents VEGF from interacting with VEGF receptors on the surface of endothelial cells (Flt-1 and KDR) and blocks the VEGF-mediated pathway, which leads to suppression of vascular endothelial cell proliferation and tumor angiogenesis. The combination of bevacizumab and atezolizumab has been approved for the treatment of renal clear cell carcinoma and the combination of bevacizumab and carboplatin for non-small cell lung cancer by the US FDA [25]. However, bevacizumab has not been approved for use in the treatment of HCC. In its phase II trial [26], bevacizumab exhibited significant clinical and biologic activity in nonmetastatic HCC, with an objective response rate of $13 \%$, a 6 -month PFS of $65 \%$, a median PFS of 6.9 months, and an OS of $53 \%$ at 1 year, $28 \%$ at 2 years, and $23 \%$ at 3 years; however, severe adverse events such as bleeding, leukopenia/ neutropenia, transient elevation of aminotransferases and hypertension occurred. No phase III trial of bevacizumab for $\mathrm{HCC}$ has been conducted to date. Another phase II study [27] led by Andrew X. Zhu showed that a combination of gemcitabine, oxaliplatin and bevacizumab (GEMOX-B regime, in which for cycle 1 (14 days), $10 \mathrm{mg} / \mathrm{kg}$ bevacizumab was administered alone intravenously on day 1 . For cycle 2 and thereafter $(28$ days/cycle), bevacizumab $10 \mathrm{mg} / \mathrm{kg}$ was administered on days 1 and 15 ; gemcitabine $1000 \mathrm{mg} / \mathrm{m} 2$ was administered as a dose rate infusion of $10 \mathrm{mg} / \mathrm{m} 2 / \mathrm{min}$ followed by oxaliplatin at $85 \mathrm{mg} / \mathrm{m} 2$ on days 2 and 16) achieved a certain effect for advanced HCC patients, with a median OS and PFS of 9.6 and 5.3 months, respectively. The GEMOX-B regime was safely administered with close monitoring and demonstrated moderate antitumor activity for patients with advanced HCC. A phase II trial of bevacizumab + erlotinib vs. sorafenib (clinicaltial.gov, No. NCT00881751) for the treatment of unresectable HCC is currently underway.

\section{Brivanib}

Brivanib is a selective dual inhibitor of VEGF and FGFR, suppressing angiogenesis and tumor cell growth. Encouraging antitumor activity has been shown in preclinical and phase I trials [28]. In a phase II, an open-label study [29] of brivanib as a first-line therapy in patients with advanced HCC, oral administration at a dose of $800 \mathrm{mg}$ once daily showed good antitumor activity, with a six-month DFS rate of $18.2 \%$, a median PFS of 2.7 months and a median OS of 10 months. Moreover, brivanib was generally well tolerated. However, the results of the subsequent randomized phase III BRISK-FL study [30] were not satisfactory, with a primary end point of OS in the brivanib-treating group that was not superior to that in the sorafenib-treated group. The median OS was 9.9 months for sorafenib and 9.5 months for brivanib. Second end point data of TTP, ORR and DCR were similar to those of sorafenib. Another multicenter, double-blind, randomized, placebo-controlled BRISK-PS study [31] showed that brivanib did not significantly improve OS compared with placebo in patients with advanced HCC and who were treated with and intolerant to sorafenib. The median OS was 9.4 months for brivanib treatment and 8.4 months for placebo treatment. Both the BRISK-FL and BRISK-PS studies failed, suggesting that brivanib does not present promising antitumor activity in advanced HCC. 


\section{Linifanib}

Linifanib is a tyrosine kinase inhibitor of VEGF and PDGFR. In the phase II trial [32] led by Han Chong Toh, administration of single-agent linifanib orally at a fasting dose of $0.25 \mathrm{mg} / \mathrm{kg}$ daily to patients with ChildPugh class A hepatic function and every other day to patients with Child-Pugh class B hepatic function showed promising clinical activity in patients with advanced HCC, with a median PFS of 3.7 months and a median OS of 9.7 months. As acceptable safety profile was also reported. In an open-label randomized phase III trial [33] conducted by Calin Cainap to evaluate the efficacy and tolerability of linifanib versus sorafenib in patients with advanced HCC, 1035 patients were randomly assigned in a 1:1 ratio to linifanib $17.5 \mathrm{mg}$ once daily or sorafenib $400 \mathrm{mg}$ twice daily. The median OS was 9.1 months in the linifanib group and 9.8 months in the sorafenib group, suggesting that linifanib and sorafenib have similar OS in advanced HCC. Indeed, the redefined superiority and noninferiority OS boundaries were not met for linifanib, and the primary end point was not reached. In addition, safety results favored sorafenib.

\section{Sunitinib}

Sunitinib is a small molecule tyrosine kinase inhibitor of VEGFR, PDGFR-a/b, c-Kit, FLT3 and RETS. The antitumor activity of sunitinib was observed in a phase II clinical trial [34] led by Andrew X. Zhu. The median OS and PFS were 9.8 months and 3.9 months, respectively, and blood vessel permeability and levels of circulating inflammatory biomarkers were altered after treatment. Sunitinib can rapidly reduce vascular leakage, especially in patients with relatively slow progression. In an open-label, phase III trial [35] evaluating whether sunitinib is superior or equivalent to sorafenib in advanced HCC, 1074 patients were stratified and randomly assigned $1: 1$ to receive sunitinib $37.5 \mathrm{mg}$ once per day or sorafenib $400 \mathrm{mg}$ twice per day. The results for sunitinib and sorafenib were as follows: median OS of 7.9 versus 10.2 months, median PFS of 3.6 versus 3.0 months, and TTP of 4.1 versus 3.8 months. Sunitinib failed to provide better anticancer activity than sorafenib but was associated with more frequent and severe adverse events (AEs). Therefore, the trial was halted in 2010. Nevertheless, sunitinib is still used in treatment for liver fibrosis due to its antiangiogenic and fibrosis inhibitory properties [36].

\section{Immunoreactive drugs}

Ipilimumab was the first real immunoreactive drug to be used clinically, and immuno-targeted medicines for malignant tumor therapy have since developed rapidly. With the recent success of checkpoint inhibitors in multiple tumors, their role in HCC has also been explored, and benefits of other immunotargeting agents can be expected when the immune checkpoint inhibitors nivolumab and pembrolizumab become available for HCC treatment.

\section{Tremelimumab}

Tremelimumab is a fully human monoclonal antibody that binds to the cytotoxic $\mathrm{T}$ lymphocyte-associated antigen 4 (CTLA-4) on the surface of activated T lymphocytes. A pilot clinical study [37] conducted by Bruon evaluated the antitumor and antiviral effects of tremelimumab in patients with advanced $\mathrm{HCC}$ and chronic $\mathrm{HCV}$ infection. After oral administration of a dose of $15 \mathrm{mg} / \mathrm{kg}$ IV every 90 days, promising anticancer and antiviral effects with a partial response rate of $17.6 \%$, a disease control rate of $76.4 \%$, time to progression of 6.48 months, and a significant decline in viral load, were observed. These findings suggest that tremelimumab immunotherapy is a promising treatment option, in particular for inhibiting the progression of hepatitis C-related advanced HCC. Tremelimumab is safe because treatment is mostly well tolerated in patients, with only a few experience disabling AEs. No patient received systemic steroids, and there were no treatment-related deaths. Another study [38] conducted by Duffy in which patients with advanced HCC were treated with tremelimumab (3.5 or $10 \mathrm{mg} / \mathrm{kg}$ IV every 4 weeks for a total of 6 doses) in combination with an ablative procedure performed during week 6 . The results show that ablative therapy induced a peripheral immune response, possibly enhancing the effect of tremelimumab in patients with advanced HCC. Six- and 12-month probabilities of tumor PFS for this refractory HCC population were 57.1 and $33.1 \%$, respectively, with a median time to TTP of 7.4 months and a median OS of 12.3 months. In addition, six-week tumor biopsies showed a clear increase in CD8+ $\mathrm{T}$ cells only in patients presenting a clinical benefit. These two studies suggested that tremelimumab treatment of patients with advanced HCC is feasible and leads to the accumulation of intratumoral $\mathrm{CD} 8+\mathrm{T}$ cells and possibly surrogate reductions in $\mathrm{HCV}$ viral load.

\section{Drugs targeting EGFR}

It is clear that overexpression of epidermal growth factor receptor (EGFR) on the membrane of HCC cells significantly promotes HCC tumorigenesis and progression. Additionally, upon ligand (EGF and TGF) binding, EGFR activates tyrosine kinases on the cell surface, which leads to the disordered growth of hepatoma cells. As a target for the treatment of HCC, several small molecule EGFR inhibitors have been developed. 


\section{Erlotinib}

Erlotinib is a tyrosine kinase inhibitor that specifically targets EGFR and EGF-1. Several studies have shown that erlotinib has good anticancer activity in non-small cell lung cancer [39] and pancreatic cancer [40]. A phase II trial [41] led by Thomas revealed that singleagent erlotinib (oral dose $150 \mathrm{mg}$ daily for 28-day cycles) is well tolerated with a modest disease-control benefit in HCC, as manifested as moderately prolonged PFS and OS when compared with historical controls. Another phase III, randomized, double-blind, placebocontrolled trial [42] led by Andrew X. Zhu revealed that erlotinib associated with sorafenib did not improve survival when compared with sorafenib plus placebo in patients with advanced HCC. The median OS in patients treated with erlotinib associated with sorafenib was 9.5 months, whereas the median OS of patients treated with sorafenib plus placebo was 8.5 months. Therefore, the efficacy of erlotinib in liver cancer remains to be further studied.

\section{Cetuximab}

Cetuximab is an IgG1 monoclonal antibody against EGFR that can specifically bind to EGFR on various tumor cells and inhibit the binding of other ligands, thereby suppressing tumor growth and progression. Cetuximab has been approved by the FDA as a firstline treatment for advanced colorectal cancer [43] and advanced head and neck cancer [44], with promising survival benefits. Unfortunately, a phase II trial [45] showed that although cetuximab could be safely administered with tolerable toxicity profiles, it exhibited no antitumor activity in HCC. Another phase II study [46], indicated that the combination of cetuximab (a dose of $400 \mathrm{mg} / \mathrm{m} 2$ initially then $250 \mathrm{mg} / \mathrm{m} 2$ weekly) and gemcitabine plus oxaliplatin (GEMOX regimen) failed to provide therapeutic effects comparable to that of either single-use cetuximab or GEMOX. Another phase II trial [47] led by Sanoff et al. showed that the combination of cetuximab and capecitabine plus oxaliplatin (capecitabine $850 \mathrm{mg} / \mathrm{m} 2$ bid days $1-14$, oxaliplatin $130 \mathrm{mg} / \mathrm{m} 2$ day 1 , and cetuximab $400 \mathrm{mg} / \mathrm{m} 2$ day 1 then $250 \mathrm{mg} / \mathrm{m} 2$ weekly for each 21 day cycle) in advanced HCC resulted in a DCR of $83 \%$, median TTP of 4.5 months and OS of 4.4 months. This result suggested that the time to progression and OS were shorter than would be expected for treatment with sorafenib.

\section{Lapatinib}

Lapatinib is another small molecule tyrosine kinase inhibitor of EGF that can effectively inhibit the ATP checkpoint and prevent homogenization and heterodimerization between EGFR and HER2, which can inhibit tumor cell growth. A multi-institutional phase II trial [48] conducted by Bekaii determined the safety and efficacy of lapatinib in advanced HCC. Interestingly, tumor and blood specimens were analyzed for expression of HER2/NEU/CEP17 and downstream signaling pathway protein status. After an oral dose of lapatinib of $1500 \mathrm{mg} /$ day in 28-day cycles, the median PFS was 1.9 months, and the median OS was 12.6 months. However, somatic mutations in EGFR (exons 18-21) and HER2/NEU were not found. In addition, PTEN, P-AKT, andP70S6 K expression did not correlate with survival. Overall, the results suggest that lapatinib is well tolerated and that only a subgroup of patients obtain a benefit, among whom the predictive molecular and clinical characteristics have not yet fully been defined.

\section{Drugs targeting the PI3K/Akt/mTOR signaling pathway}

The PI3K/Akt/mTOR signaling pathway plays an important role in HCC tumorigenesis and progression. PI3K regulates the proliferation, growth, survival and angiogenesis of tumor cells. Activated PI3K phosphorylates and activates AKT, which is localized in the plasma membrane. AKT transmits the signal to downstream targets and then activates mTOR. Blocking this signaling pathway, particularly inhibition of mTOR activation, may specifically suppress tumor cell growth.

\section{Sirolimus}

Sirolimus is an inhibitor of mTOR [49]. By inhibiting expression of hypoxia inducible factor-1 $\alpha$ and decreasing the synthesis and secretion of VEGF, then effectively inhibits angiogenesis and HCC proliferation. The PFS and OS of patients after treatment with rapamycin analogs (sirolimus) was 15.3 weeks and 26.4 weeks in a phase II trial [50] led by Decaens. One patient achieved a complete response (CR), with 8 having stable disease (SD); a median OS of 6.5 months was reported in another trial led by Rizell [51]. Both studies suggest that first-line sirolimus has antitumoural efficacy in advanced HCC. However, there are no reports of phase III trials of sirolimus, and larger trials with Child-Pugh A patients are needed.

\section{Everolimus}

Everolimus is a rapalog and inhibitor of mTOR that has been approved for the treatment of renal and breast cancer. The results from a phase I/II clinical trial [52] preliminarily revealed the curative effect of everolimus for HCC, with a median OS and PFS of 8.4 and 3.8 months, respectively. Considering the different targets of everolimus and sorafenib, Andrew X. Zhu led the randomized EVOLVE-1 trial [53] with HCC patients who were not treated with sorafenib. The results, 
however, fell short of expectations. The everolimus group showed a median PFS of only 7.6 months and a median OS of 3.0 months. Moreover, OS was not improved after treatment with everolimus among patients with liver cancer for whom sorafenib was ineffective or who were intolerant of sorafenib. Everolimus has also been evaluated in a phase III study as a second-line treatment for $\mathrm{HCC}$, though with negative results in an unselected patient population [54]. Thus far, everolimus has not yet been FDA approved for the treatment of HCC.

\section{C-met inhibitors}

C-Met is a proto-oncogene, and the protein becomes phosphorylated upon binding of hepatocyte growth factor. This phosphorylation activates a series of downstream signaling pathways, leading to cell proliferation and survival, cytoskeleton reorganization, cell migration and invasion, and vascular regeneration. This pathway is closely related to the occurrence and development of tumor, and the growth of tumor cells can be inhibited by inhibiting expression of c-Met. Indeed, a previous study demonstrated that overexpression of c-Met is an independent risk factor for poor prognosis in $\mathrm{HCC}$ patients [55]. Therefore, c-Met may constitute an alternative molecular target for the development of advanced HCC therapy.

\section{Tivantinib (ARQ197)}

Tivantinib is an effective small molecule c-Met receptor tyrosine kinase inhibitor. In a phase II trial [56], patients with advanced HCC and Child-Pugh A liver function in the c-Met high-expression group received second-line tivantinib therapy and exhibited a PFS of 2.7 months, which was significantly longer than the 1.4 months observed in the placebo group. Furthermore, Rimassa et al. carried out a phase III study [57] to evaluate the efficacy and safety of tivantinib as a second-line therapy for HCC patients with high Met expression. According to the results, the OS in the tivantinib group was 8.4 months, which was lower than that in the placebo group (9.1 months), and tivantinib-treated patients were more prone to severe adverse events. Overall, tivantinib failed to improve the OS of advanced HCC patients with high Met expression after sorafenib treatment.

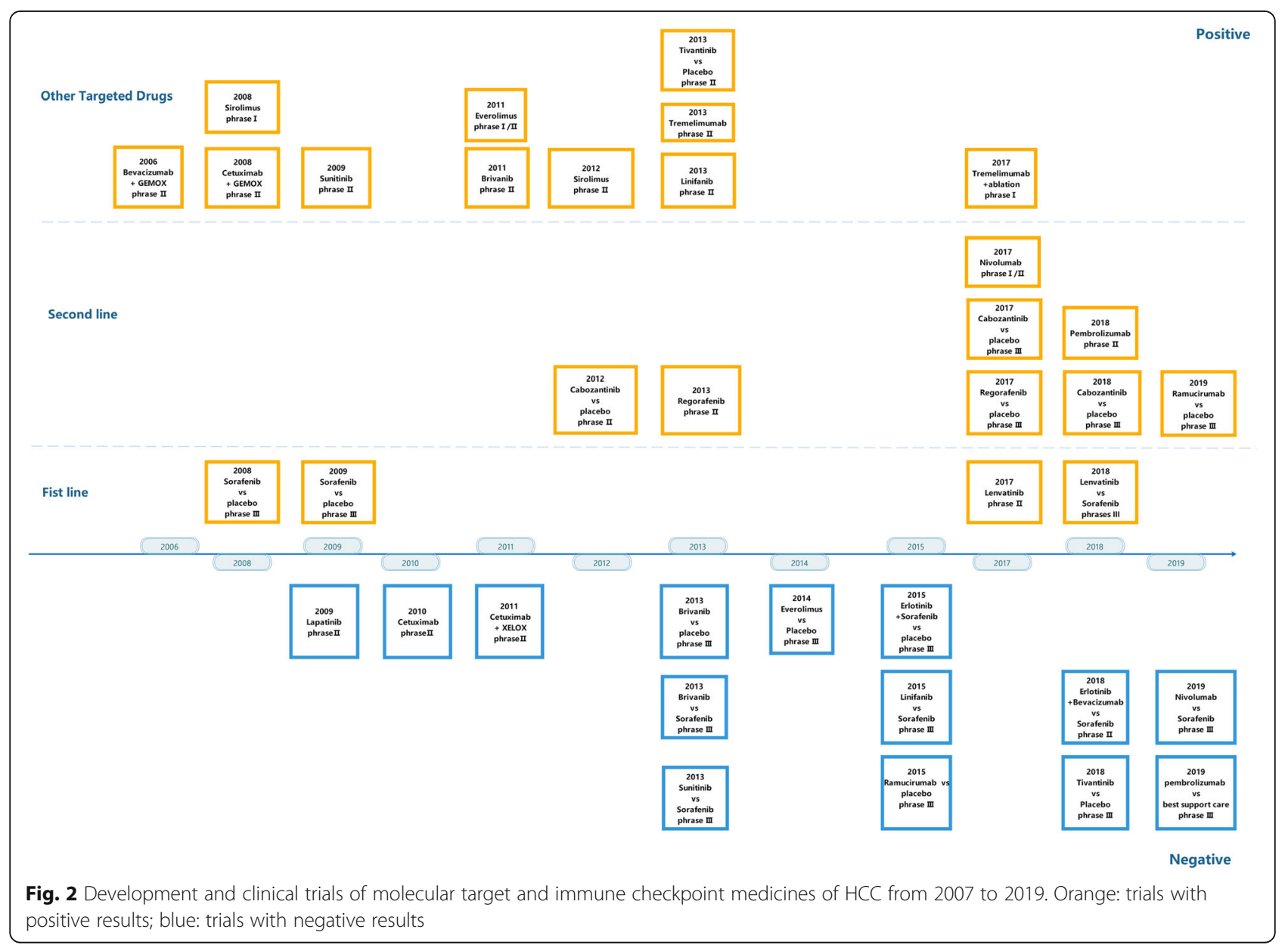




\section{Conclusions and future expectations}

Systematic treatment for advanced HCC has changed drastically in the past decade since the introduction of sorafenib as the first small molecule targeting agent in 2007 [58] (Fig. 2). As the pathways and targets closely related to the tumorigenesis and progression of $\mathrm{HCC}$ have been revealed, novel molecular targeted therapy agents are constantly being developed and tested, with great expectations of treatment for advanced HCC. However, almost every test of many of these molecular targeted agents during the 10-year period from 2007 to 2016 failed due to a low response rate and high toxicity in phase II or phase III clinical trials. Nonetheless, it is encouraging that in the past 2 years (2017 through 2018), four novel drugs-lenvatinib, regorafenib, cabozantinib, and ramucirumab-have successfully emerged from clinical trials and been recommended for clinical use as alternative or supplements to sorafenib [59]. As recommended by updated BCLC treatment algorithms, lenvatinib is now feasible as an alternative to sorafenib as a first-line treatment for advanced HCC in clinical practice. Regorafenib, cabozantinib, and ramucirumab are appropriate supplements for sorafenib as second-line treatments for patients with advanced $\mathrm{HCC}$ who are resistant, have progressed or do not tolerate sorafenib. Recently, with promising outcomes revealed from phase II trials, immune PD-1/PD-L1 checkpoint inhibitors such as nivolumab and pembrolizumab have been applied for HCC treatment [60]. However, in phase III trials, the primary endpoints of OS improvement with nivolumab and pembrolizumab were not statistically significant. Thus, immune PD-1/PD-L1 checkpoint therapy remains to be further investigated.

Finally, the great progress in the number of molecular targeted therapies and immune checkpoint therapy options for advanced HCC will benefit many patients, likely rendering drug selection and sequences challenging. First, combination therapy using targeted treatments with immune checkpoint inhibitors is expected to yield even better effects when these drugs eventually become available. In addition, these new drugs or combination therapies may benefit a wide range of patients in early, intermediate and even advanced stages of HCC as an adjuvant to improve the response rate of chemotherapy, TACE and radiotherapy, to downstage unresectable HCC or to suppress recurrence with high risk. Moreover, biomarkers and alternative predictors, including conventional tumor markers, precise checkpoint targets or pathways, tumor mutational burden (TMB) and circulation tumor cells, remain to be further investigated for precisely identifying patients for appropriate treatment.

\section{Abbreviations}

BCLC: Barcelona Clinical Liver cancer; C-MET: Hepatocyte growth factor receptor; CTLA-4: Ytotoxic T lymphocyte associated antigen 4; DCR: Disease control rate; EGFR: Epidermal growth factor receptor; FDA: Food and drug administration; FGFR: Fibroblast growth factor receptor; GEMOX-

B: Gemcitabine combined with oxaliplatin and bevacizumab; HCC: Hepatocellular carcinoma; KIT: Stem cell factor receptor; OS: Overall survival; PD-1: Programmed death-1; PDGFR: Platelet-derived growth factor receptor; PD-L1: Programmed death-ligand 1; PFS: Progression-free survival; RET: Glial cell-derived neurotrophic factor receptor; TACE: Transarterial chemoembolization; Tie2: Angiopoietin receptor; TMB: Tumor mutational burden; TTP: Time to progression; VEGFR: Vascular endothelial growth factor receptor

\section{Acknowledgments}

Not applicable.

\section{Authors' contributions}

All the authors contributed in the preparation of this work. ZYL and $Y L$ were drafted and revised the article; $R L$ and JZY were responsible for the theme, final editing, and preparation of the manuscript for submission; JYZ, YMZ, $Y Q L, Z H L, Q L$ and ML critically revised the manuscript. All authors read and approved the final manuscript.

\section{Funding}

This reseach was supported by Regional science fund project of China natural science foundation (NO.81660498);

Youth talent fund project of China natural science foundation (NO. 81803007);

Youth talent fund project of GuangXi natural science foundation (NO. 2016GXNSFBA380090, 2018GXNSFBA281030, 2018GXNSFBA281091);

Guangxi Medical and Health Appropriate Technology Development and Application Project (No. S2017101, S2018062);

The Basic Ability Enhancement Program for Young and Middle-aged Teachers of Guangxi (2017KY0120),

Guangxi Scholarship Fund of Guangxi Education Department.

\section{Availability of data and materials}

Not applicable.

Ethics approval and consent to participate

Not applicable.

\section{Consent for publication}

Not applicable.

\section{Competing interests}

The authors declare that they have no competing interests.

\section{Author details}

'School of Oncology, Guangxi Medical University, Nanning, Guangxi 530021, People's Republic of China. ${ }^{2}$ Department of Medical Oncology, Guangxi Medical University Cancer Hospital, Nanning, Guangxi 530021, People's Republic of China. ${ }^{3}$ Department of Hepatobiliary Surgery, Guangxi Medical University Cancer Hospital, Nanning, Guangxi 530021, People's Republic of China.

Received: 18 March 2019 Accepted: 4 September 2019 Published online: 04 November 2019

\section{References}

1. Llovet JM, Zucman-Rossi J, Pikarsky E, et al. Hepatocellular carcinoma. Nat Rev Dis Primers. 2016;2:16018.

2. Llovet JM, Ricci S, Mazzaferro V, Hilgard P, Gane E, Blanc JF, de Oliveira AC, Santoro A, Raoul JL, Forner A, et al. Sorafenib in advanced hepatocellular carcinoma. N Engl J Med. 2008;359(4):378-90.

3. Cheng AL, Kang YK, Chen Z, Tsao CJ, Qin S, Kim JS, Luo R, Feng J, Ye S, Yang TS, et al. Efficacy and safety of sorafenib in patients in the Asia-Pacific region with advanced hepatocellular carcinoma: a phase III randomised, double-blind, placebo-controlled trial. Lancet Oncol. 2009;10(1):25-34.

4. Elkhoueiry AB, Sangro B, Yau T, Crocenzi TS, Kudo M, Hsu C, Kim TY, Choo SP, Trojan J, Rd WT. Nivolumab in patients with advanced hepatocellular carcinoma (CheckMate 040): an open-label, non-comparative, phase 1/2 dose escalation and expansion trial. Lancet. 2017;389(10088):2492. 
5. Finn R, Chan SL, Zhu AX, Knox J, Cheng A, Siegel A, Bautista O, Watson PA Kudo M. Pembrolizumab vs best supportive care for second-line advanced hepatocellular carcinoma: Randomized, phase 3 KEYNOTE-240 study. J Clin Oncol. 2016;35(4_suppl):TPS503-503TPS503.

6. Tai WT, Cheng AL, Shiau CW, Huang HP, Huang JW, Chen PJ, Chen KF. Signal transducer and activator of transcription 3 is a major kinaseindependent target of sorafenib in hepatocellular carcinoma. J Hepatol. 2011:55(5):1041-8.

7. Stotz M, Gerger A, Haybaeck J, Kiesslich T, Bullock MD, Pichler M. Molecula targeted therapies in hepatocellular carcinoma: past, Present and Future. Anticancer Res. 2015;35(11):5737-44.

8. Forner A, Reig ME, de Lope CR, Bruix J. Current strategy for staging and treatment: the BCLC update and future prospects. Semin Liver Dis. 2010; 30(1):61-74

9. Benson AB 3rd, Abrams TA, Ben-Josef E, Bloomston PM, Botha JF, Clary BM, Covey A, Curley SA, D'Angelica MI, Davila R, et al. NCCN clinical practice guidelines in oncology: hepatobiliary cancers. J Natl Compr Canc Netw. 2009;7:350-91.

10. Capozzi M, De Divitiis C, Ottaiano A, von Arx C, Scala S, Tatangelo F, Delrio $P$, Tafuto S. Lenvatinib, a molecule with versatile application: from preclinical evidence to future development in anti-cancer treatment. Cancer Manag Res. 2019;11:3847-60.

11. Brose MS, Worden FP, Newbold KL, Guo M, Hurria A. Effect of age on the efficacy and safety of Lenvatinib in radioiodine-refractory differentiated thyroid Cancer in the phase III SELECT trial. J Clin Oncol. 2017;35(23):2692-9.

12. Ikeda K, Kudo M, Kawazoe S, Osaki Y, Ikeda M, Okusaka T, Tamai T, Suzuki T, Hisai T, Hayato S, et al. Phase 2 study of lenvatinib in patients with advanced hepatocellular carcinoma. J Gastroenterol. 2017;52(4):512-9.

13. Kudo M, Finn RS, Qin S, Han KH, Ikeda K, Piscaglia F, Baron A, Park JW, Han $G$, Jassem J, et al. Lenvatinib versus sorafenib in first-line treatment of patients with unresectable hepatocellular carcinoma: a randomised phase 3 non-inferiority trial. Lancet. 2018;391(10126):1163-73.

14. Benson AB, D'Angelica Ml, Abbott DE, Abrams TA, Alberts SR, Anaya DA, Anders R, Are C, Brown D, Chang DT, et al. Guidelines insights: hepatobiliary cancers, version 2.2019. J Natl Compr Cancer Netw. 2019;17(4):302-10.

15. Bruix J, Tak WY, Gasbarrini A, Santoro A, Colombo M, Lim HY, Mazzaferro V, Wiest R, Reig M, Wagner A. Regorafenib as second-line therapy for intermediate or advanced hepatocellular carcinoma: multicentre, open-label, phase II safety study $i \vec{s}$. Eur J Cancer. 2013;49(16):3412-9.

16. Bruix J, Qin S, Merle P, Granito A, Huang YH, Bodoky G, Pracht M, Yokosuka O, Rosmorduc O, Breder V, et al. Regorafenib for patients with hepatocellular carcinoma who progressed on sorafenib treatment (RESORCE): a randomised, double-blind, placebo-controlled, phase 3 trial. Lancet. 2017;389(10064):56-66.

17. Benson AB 3rd, D'Angelica MI, Abbott DE, Abrams TA, Alberts SR, Saenz DA, Are C, Brown DB, Chang DT, Covey AM, et al. NCCN guidelines insights: hepatobiliary cancers, version 1.2017. J Natl Compr Cancer Netw. 2017;15(5): 563-73.

18. Kelley RK, Verslype C, Cohn AL, Yang TS, Su WC, Burris H, Braiteh F, Vogelzang N, Spira A, Foster P. Cabozantinib in hepatocellular carcinoma: results of a phase 2 placebo-controlled randomized discontinuation study. Ann Oncol. 2017;28(3):528-34.

19. Abou-Alfa GK, Meyer T, Cheng AL, El-Khoueiry AB, Rimassa L, Ryoo BY, Cicin I, Merle P, Chen Y, Park JW. Cabozantinib in patients with advanced and progressing hepatocellular carcinoma. N Engl J Med. 2018;379(1):54.

20. Fuchs CS, Tomasek J, Yong CJ, Dumitru F, Passalacqua R, Goswami C, Safran H, Dos Santos LV, Aprile G, Ferry DR, et al. Ramucirumab monotherapy for previously treated advanced gastric or gastro-oesophageal junction adenocarcinoma (REGARD): an international, randomised, multicentre, placebo-controlled, phase 3 trial. Lancet. 2014;383(9911):31-9.

21. Ciuleanu TE, Arrieta O, Prabhash K, Syrigos KN, Göksel T, Park K, Kowalyszyn RD, Pikiel J, Czyzewicz G, Orlov S. REVEL: A randomized, double-blind, phase III study of docetaxel (DOC) and ramucirumab (RAM; IMC-1121B) versus DOC and placebo (PL) in the second-line treatment of stage IV non-small cell lung cancer (NSCLC) following disease progression after one prior pla. J Clin Oncol Off J Am Soc Clin Oncol. 2014;32(18_suppl):LBA8006.

22. Zhu AX, Park JO, Ryoo BY, Yen CJ, Poon R, Pastorelli D, Blanc JF, Chung HC, Baron AD, Pfiffer TE, et al. Ramucirumab versus placebo as second-line treatment in patients with advanced hepatocellular carcinoma following first-line therapy with sorafenib $(\mathrm{REACH})$ : a randomised, double-blind, multicentre, phase 3 trial. Lancet Oncol. 2015;16(7):859-70.
23. Zhu AX, Kang YK, Yen CJ, Finn RS, Galle PR, Llovet JM, Assenat E, Brandi G, Pracht M, Lim HY, et al. Ramucirumab after sorafenib in patients with advanced hepatocellular carcinoma and increased a-fetoprotein concentrations (REACH-2): a randomised, double-blind, placebo-controlled, phase 3 trial. Lancet Oncol. 2019;20(2):282-96.

24. Zhu AX, Finn RS, Edeline J, Cattan S, Ogasawara S, Palmer D, Verslype C, Zagonel V, Fartoux L, Vogel A. Pembrolizumab in patients with advanced hepatocellular carcinoma previously treated with sorafenib (KEYNOTE-224): a non-randomised, open-label phase 2 trial. Lancet Oncol. 2018;19(7):940-52.

25. Larkin J, Paine A, Foley G, Mitchell S, Chen C. First-line treatment in the management of advanced renal cell carcinoma: systematic review and network meta-analysis. Expert Opin Pharmacother. 2015;16(13):1915-27.

26. Siegel AB, Cohen El, Ocean A, Lehrer D, Goldenberg A, Knox JJ, Chen H, Clarkgarvey S, Weinberg A, Mandeli J. Phase II trial evaluating the clinical and biologic effects of bevacizumab in Unresectable hepatocellular carcinoma. J Clin Oncol. 2008;26(18):2992-8.

27. Zhu AX, Blaszkowsky LS, Ryan DP, Clark JW, Muzikansky A, Horgan K, Sheehan S, Hale KE, Enzinger PC, Bhargava P. Phase II study of gemcitabine and oxaliplatin in combination with bevacizumab in patients with advanced hepatocellular carcinoma. J Clin Oncol. 2006;24(12):1898-903.

28. Jonker DJ, Rosen LS, Sawyer MB, Braud FD, Wilding G, Sweeney CJ, Jayson GC, Mcarthur GA, Rustin G, Goss G. A phase I study to determine the safety, pharmacokinetics and pharmacodynamics of a dual VEGFR and FGFR inhibitor, brivanib, in patients with advanced or metastatic solid tumors. Ann Oncol. 2011;22(6):1413.

29. Park JW, Finn RS, Kim JS, Karwal M, Li RK, Ismail F, Thomas M, Harris R, Baudelet C, Walters I, et al. Phase II, open-label study of brivanib as first-line therapy in patients with advanced hepatocellular carcinoma. Clin Cancer Res. 2011;17(7):1973-83.

30. Johnson PJ, Qin S, Park JW, Poon RT, Raoul JL, Philip PA, Hsu CH, Hu TH, Heo J, Xu J, et al. Brivanib versus sorafenib as first-line therapy in patients with unresectable, advanced hepatocellular carcinoma: results from the randomized phase III BRISK-FL study. J Clin Oncol. 2013;31(28):3517-24.

31. Llovet JM, Decaens T, Raoul JL, Boucher E, Kudo M, Chang C, Kang YK, Assenat E, Lim HY, Boige V. Brivanib in patients with advanced hepatocellular carcinoma who were intolerant to Sorafenib or for whom Sorafenib failed: results from the randomized phase III BRISK-PS study. J Clin Oncol. 2013;31(28):3509.

32. Toh HC, Chen PJ, Carr BI, Knox JJ, Gill S, Ansell P, McKeegan EM, Dowell B, Pedersen M, Qin Q, et al. Phase 2 trial of linifanib (ABT-869) in patients with unresectable or metastatic hepatocellular carcinoma. Cancer. 2013;119(2): 380-7.

33. Cainap C, Qin S, Huang WT, Chung IJ, Pan H, Cheng Y, Kudo M, Kang YK, Chen PJ, Toh HC. Linifanib versus Sorafenib in patients with advanced hepatocellular carcinoma: results of a randomized phase III trial. J Clin Oncol. 2015;33(2):172-9.

34. Zhu AX, Sahani DV, Duda DG, di Tomaso E, Ancukiewicz M, Catalano OA, Sindhwani V, Blaszkowsky LS, Yoon SS, Lahdenranta J, et al. Efficacy, safety, and potential biomarkers of sunitinib monotherapy in advanced hepatocellular carcinoma: a phase II study. J Clin Oncol. 2009:27(18):3027-35.

35. Cheng AL, Kang YK, Lin DY, Park JW, Kudo M, Qin S, Chung HC, Song X, Xu J, Poggi $G$, et al. Sunitinib versus sorafenib in advanced hepatocellular cancer: results of a randomized phase III trial. J Clin Oncol. 2013;31(32):4067-75.

36. Majumder S, Piguet AC, Dufour JF, Chatterjee S. Study of the cellular mechanism of Sunitinib mediated inactivation of activated hepatic stellate cells and its implications in angiogenesis. Eur J Pharmacol. 2013 705(1-3):86-95.

37. Sangro B, Gomezmartin C, de la Mata M, Iñarrairaegui M, Garralda E, Barrera P, Riezuboj Jl, Larrea E, Alfaro C, Sarobe P. A clinical trial of CTLA-4 blockade with tremelimumab in patients with hepatocellular carcinoma and chronic hepatitis C. J Hepatol. 2013;59(1):81-8.

38. Duffy AG, Ulahannan SV, Makorova-Rusher O, Rahma O, Wedemeyer H, Pratt D, Davis JL, Hughes MS, Heller T, ElGindi M, et al. Tremelimumab in combination with ablation in patients with advanced hepatocellular carcinoma. J Hepatol. 2017;66(3):545-51.

39. Greenhalgh J, Bagust A, Boland A, Dwan K, Beale S, Hockenhull J, Proudlove C, Dundar Y, Richardson M, Dickson R, et al. Erlotinib and gefitinib for treating non-small cell lung cancer that has progressed following prior chemotherapy (review of NICE technology appraisals 162 and 175): a systematic review and economic evaluation. Health Technol Assess. 2015. 19(47):1-134. 
40. Furuse J, Gemma A, Ichikawa W, Okusaka T, Seki A, Ishii T. Postmarketing surveillance study of erlotinib plus gemcitabine for pancreatic cancer in Japan: POLARIS final analysis. Jpn J Clin Oncol. 2017;47(9):832-9.

41. Thomas MB, Garrett-Mayer E, Anis M, Anderton K, Bentz T, Edwards A, Brisendine A, Weiss $G$, Siegel $A B$, Bendell J, et al. A randomized phase II open-label multi-institution study of the combination of bevacizumab and Erlotinib compared to Sorafenib in the first-line treatment of patients with advanced hepatocellular carcinoma. Oncology. 2018;94(6):329-39.

42. Zhu AX, Rosmorduc O, Evans TR, Ross PJ, Santoro A, Carrilho FJ, Bruix J, Qin S, Thuluvath PJ, Llovet JM. SEARCH: a phase III, randomized, double-blind, placebo-controlled trial of sorafenib plus erlotinib in patients with advanced hepatocellular carcinoma. J Clin Oncol. 2015;33(6):559-66.

43. Miyamoto Y, Suyama K, Baba H. Recent advances in targeting the EGFR signaling pathway for the treatment of metastatic colorectal Cancer. Int J Mol Sci. 2017;18(4):752

44. Merlano M, Occelli M. Review of cetuximab in the treatment of squamous cell carcinoma of the head and neck. Ther Clin Risk Manag. 2007;3(5):871-6.

45. Zhu AX. Beyond sorafenib: novel targeted therapies for advanced hepatocellular carcinoma. Expert Opin Investig Drugs. 2010;19(5):663-72.

46. Asnacios A, Fartoux L, Romano O, Tesmoingt C, Louafi SS, Mansoubakht T, Artru P, Poynard T, Rosmorduc O, Hebbar M, et al. Gemcitabine plus oxaliplatin (GEMOX) combined with cetuximab in patients with progressive advanced stage hepatocellular carcinoma: results of a multicenter phase 2 study. Cancer. 2008;112(12):2733-9.

47. Sanoff HK, Bernard S, Goldberg RM, Morse MA, Garcia R, Woods L, Moore DT, O'Neil BH. Phase II study of Capecitabine, Oxaliplatin, and Cetuximab for advanced hepatocellular carcinoma. Gastrointest Cancer Res. 2011:4(3):78-83.

48. Bekaii-Saab T, Markowitz J, Prescott N, Sadee W, Heerema N, Wei L, Dai Z, Papp A, Campbell A, Culler K, et al. A multi-institutional phase II study of the efficacy and tolerability of lapatinib in patients with advanced hepatocellular carcinomas. Clin Cancer Res. 2009;15(18):5895-901.

49. Meng LH, Zheng XF. Toward rapamycin analog (rapalog)-based precision cancer therapy. Acta Pharmacol Sin. 2015;36(10):1163-9.

50. Decaens T, Al E. Phase II study of sirolimus in treatment-naive patients with advanced hepatocellular carcinoma. Dig Liver Dis. 2012;44(7):610-6.

51. Rizell $M$, Andersson $M$, Cahlin $C$, Hafström L, Olausson M, Lindnér P. Effects of the MTOR inhibitor sirolimus in patients with hepatocellular and cholangiocellular cancer. Int J Clin Oncol. 2008;13(1):66.

52. Zhu AX, Abrams TA, Miksad R, Blaszkowsky LS, Meyerhardt JA, Zheng H, Muzikansky A, Clark JW, Kwak EL, Schrag D. Phase 1/2 study of everolimus in advanced hepatocellular carcinoma. Cancer. 2011;117(22):5094-102.

53. Zhu AX, Kudo M, Assenat E, Cattan S, Kang YK, Lim HY, Poon RTP, Blanc JF, Vogel A, Chen CL. Effect of Everolimus on survival in advanced hepatocellular carcinoma after failure of Sorafenib: the EVOLVE-1 randomized clinical trial. J Am Med Assoc. 2014;312(1):57-67.

54. Llovet JM, Montal R, Sia D, Finn RS. Molecular therapies and precision medicine for hepatocellular carcinoma. Nat Rev Clin Oncol. 2018;15(10): 599-616.

55. Ke AW, Shi GM, Zhou J, Wu FZ, Ding ZB, Hu MY, Xu Y, Song ZJ, Wang ZJ, $\mathrm{Wu} \mathrm{JC}$, et al. Role of overexpression of CD151 and/or c-met in predicting prognosis of hepatocellular carcinoma. Hepatology. 2009;49(2):491-503.

56. Santoro A, Rimassa L, Borbath I, Daniele B, Salvagni S, Van Laethem JL, Van Vlierberghe $H$, Trojan J, Kolligs FT, Weiss A, et al. Tivantinib for second-line treatment of advanced hepatocellular carcinoma: a randomised, placebocontrolled phase 2 study. Lancet Oncol. 2013;14(1):55-63.

57. Rimassa L, Assenat E, Peck-Radosavljevic M, Pracht M, Zagonel V, Mathurin P, Rota Caremoli E, Porta C, Daniele B, Bolondi L, et al. Tivantinib for secondline treatment of MET-high, advanced hepatocellular carcinoma (METIVHCC): a final analysis of a phase 3, randomised, placebo-controlled study. Lancet Oncol. 2018:19(5):682-93.

58. Kudo M. Targeted and immune therapies for hepatocellular carcinoma: predictions for 2019 and beyond. World J Gastroenterol. 2019;25(7):789-807.

59. Kudo M. Systemic Therapy for Hepatocellular Carcinoma: Latest Advances. Cancers. 2018;10(11):412.

60. Lee RC, Tebbutt N. Systemic treatment of advanced hepatocellular cancer: new hope on the horizon. Expert Rev Anticancer Ther. 2019;19(4):343-53.

\section{Publisher's Note}

Springer Nature remains neutral with regard to jurisdictional claims in published maps and institutional affiliations.

\section{Ready to submit your research? Choose BMC and benefit from}

- fast, convenient online submission

- thorough peer review by experienced researchers in your field

- rapid publication on acceptance

- support for research data, including large and complex data types

- gold Open Access which fosters wider collaboration and increased citations

- maximum visibility for your research: over $100 \mathrm{M}$ website views per year

At $\mathrm{BMC}$, research is always in progress.

Learn more biomedcentral.com/submissions 